\title{
Subacute Cutaneous Lupus Erythematosus-Like Eruption Induced by Durvalumab: A Case Report and Literature Review
}

\author{
Nathathai Pratumchart' \\ Kumutnart Chanprapaph (iD) \\ Nuttapong Topibulpong ${ }^{2}$ \\ Jutamas Tankunakorn (D) \\ 'Division of Dermatology, Department of \\ Internal Medicine, Faculty of Medicine, \\ Ramathibodi Hospital, Mahidol \\ University, Bangkok, Thailand; ${ }^{2}$ Division \\ of Oncology, Department of Internal \\ Medicine, Faculty of Medicine, \\ Ramathibodi Hospital, Mahidol \\ University, Bangkok, Thailand
}

\begin{abstract}
Reports of immune-related adverse events caused by programmed cell deathligand 1 (PD-L1) inhibitor have been emerging. Herein, we report a subacute cutaneous lupus erythematosus (SCLE)-like eruption presented after the treatment of durvalumab in a patient with extensive-stage small cell lung carcinoma. A 74-year-old Thai man was referred to our department after experiencing multiple dusky red to brownish papules and patches with scale and erosions on photo-distributed areas after receiving 3 infusion cycles of durvalumab. Histological finding revealed epidermal atrophy with interface changes and superficial perivascular infiltration of lymphocytes. Serum antinuclear antibodies (ANA) was 1:320 and anti-Ro/Sjogren's-syndrome-related antigen A (anti-Ro/SSA) antibodies were positive $(2+)$. Based on the history and clinicopathological correlation, the diagnosis of SCLE-like eruption due to durvalumab was made. To the best of our knowledge, this is the first case of durvalumab-induced SCLE.
\end{abstract}

Keywords: programmed cell death-ligand 1 inhibitor, durvalumab, subacute cutaneous lupus erythematosus, immune-related adverse events

\section{Introduction}

Immune checkpoint inhibitors (ICIs), which target cytotoxic T-lymphocyte-associated protein-4 (CTLA-4), programmed cell death 1(PD-1) and programmed cell death-ligand 1 (PD-L1), have been established as a dominant paradigm in management of advanced malignancies. Blocking specific molecular targets inhibits cancer cell growth, progression, and spread, often resulting in long-term survival. However, by increasing the activity of the immune system, these blockades can lead to inflammatory effects, which are often termed immune-related adverse events. ${ }^{1}$ These events have a significant impact on patient's quality of life and may impair ICIs treatment efficacy due to ICIs dose-limiting effects. Immunerelated cutaneous adverse events (irCAEs) are the most common and earliest to occur in ICIs patients; thus, understanding their clinicopathologic features and effective management strategies is critical to a successful oncodermatologic practice. ${ }^{2}$ Recently, cutaneous connective tissue disease-like eruptions have been observed in patients receiving ICIs, with subacute cutaneous lupus erythematosus (SCLE) being the most common manifestation. ${ }^{3}$

The newly developed anti-PD-L1 agents also have emerged in drug-related cutaneous adverse events. Regarding the National Comprehensive Cancer Network (NCCN) guidelines, durvalumab is a PD-L1 blocker which has been 
approved by United State FDA in 2020 as a first-line treatment for adult patients with extensive-stage small cell lung carcinoma (ES-SCLC) in combination with platinum-etoposide as a result of the Phase III CASPIAN trial. $^{2}$

\section{Case Report}

A 74-year-old male with no known underlying disease developed chronic productive cough for 9 months. He had history of heavy smoking (50 pack-years) for several decades. The initial chest radiography revealed a lung mass. His computerized tomography (CT) demonstrated a large lobulated lung mass at the right lower lobe with surrounding reticulonodular opacities. A transbronchial lung biopsy was performed and revealed SCLC on histopathology. He also had leptomeningeal and ipsilateral pleural metastasis. As a result, the diagnosis of ES-SCLC was made. The oncologist began treatment with standardof-care chemotherapies including carboplatin and etoposide. One month later, he received the second cycle of standard chemotherapies plus durvalumab (Imfinzi ${ }^{\circledR}$ ). The therapeutic agents were administered intravenously once a month with no dose adjustments. Before the fourth cycle of treatment (after receiving 3 infusion cycles of durvalumab), he noticed asymptomatic erythematous skin lesions on the extensor surface of his forearms and back. He frequently engaged in outdoor activities with insufficient photoprotection. Three weeks after the fourth cycle, the skin eruptions worsened, becoming darker and more widespread on his face, neck, trunk, and extensor surface of upper extremities. Dermatologic consultation was made. Physical examination showed multiple dusky red to brownish papules and patches covered by scales and crusts with some erosions predominately on sun-exposed areas (face, upper chest, back, dorsum of both forearms) and spared the mucosa (Figure 1A-D). Nikolsky's sign was positive. A punch biopsy obtained from the lesion on the back revealed a superficial perivascular infiltration and epidermal atrophy with marked interface change (Figure 2A). Remarkably, thin epidermis was necrosed and separated with few dysmaturation of atypical basal keratinocytes (Figure 2B). ANA was detected by using indirect immunofluorescence antibody (IFA) technique showing a titer of 1:320 with a fine speckled pattern. Anti-Ro/SSA was positive (2+) while anti-La/SSB and anti-histone were negative. The erythrocyte sedimentation rate (ESR) and C-reactive protein (CRP) levels were elevated. Durvalumab-induced SCLE was diagnosed in our patient based on history, clinicopathological and serological findings. There was no evidence of systemic lupus erythematosus.

Because the skin lesions progressed to grade 3 (covering more than $30 \%$ of body surface area [BSA] with moderate pruritus) according to the Common Terminology Criteria for Adverse Events (CTCAE) version $5.0,{ }^{3}$ all chemotherapies and durvalumab were discontinued. The patient received $1 \mathrm{mg} / \mathrm{kg} / \mathrm{day}$ of oral prednisolone for the first 2 weeks, followed by hydroxychloroquine (HCQ) $200 \mathrm{mg}$ daily and topical $1.25 \%$ hydrocortisone ointment twice daily. Due to significant clinical improvement, topical and systemic corticosteroid was discontinued in the next 2 weeks. Overall, the skin lesions resolved with post-inflammatory hyperpigmentation within one month of drug withdrawal (Figure 3AD). Furthermore, the patient was advised to undergo strict photo-protection. The standard chemotherapies were resumed without durvalumab and the same hydroxychloroquine dose was maintained for 5 months. During the fifth and sixth cycles of chemotherapies, no new lesions appeared. A CT chest follow-up after 6 months of chemotherapies revealed a reduction in the size of right lung mass with a slightly increased small right pleural effusion and nodular pleural thickening. Unfortunately, the patient failed to follow-up with the oncologist after receiving the sixth cycle of chemotherapies. He passed away 3 months later due to disease progression with no cutaneous eruption recurrence.

\section{Discussion}

Checkpoint inhibitors are monoclonal antibodies that block CTLA-4, PD-1, or PD-L1 leading to anti-tumor immune response. They can cause a wide range of side effects, most of which are immune-related adverse events that manifest in the gastrointestinal tract, liver, and skin, though any organ can be affected. The diverse groups of cutaneous reactions have been implicated in checkpoint inhibitions. Maculopapular rash, pruritus, psoriasiform and lichenoid eruptions are the most prevalent subtypes. ${ }^{3}$ Immune-related adverse events occur in $70 \%$ of patients treated with PD-1 or PD-1 inhibitor, typically in cutaneous system. Bullous pemphigoid-like, vitiligo-like, and psoriasiform eruptions are frequently reported. ${ }^{3}$

A large retrospective cohort study found that only $0.025 \%$ of patients developed skin lesions resembling de novo cutaneous connective tissue disease. Median time to onset of skin lesions was 8 months (0.5-26). SCLE is the 

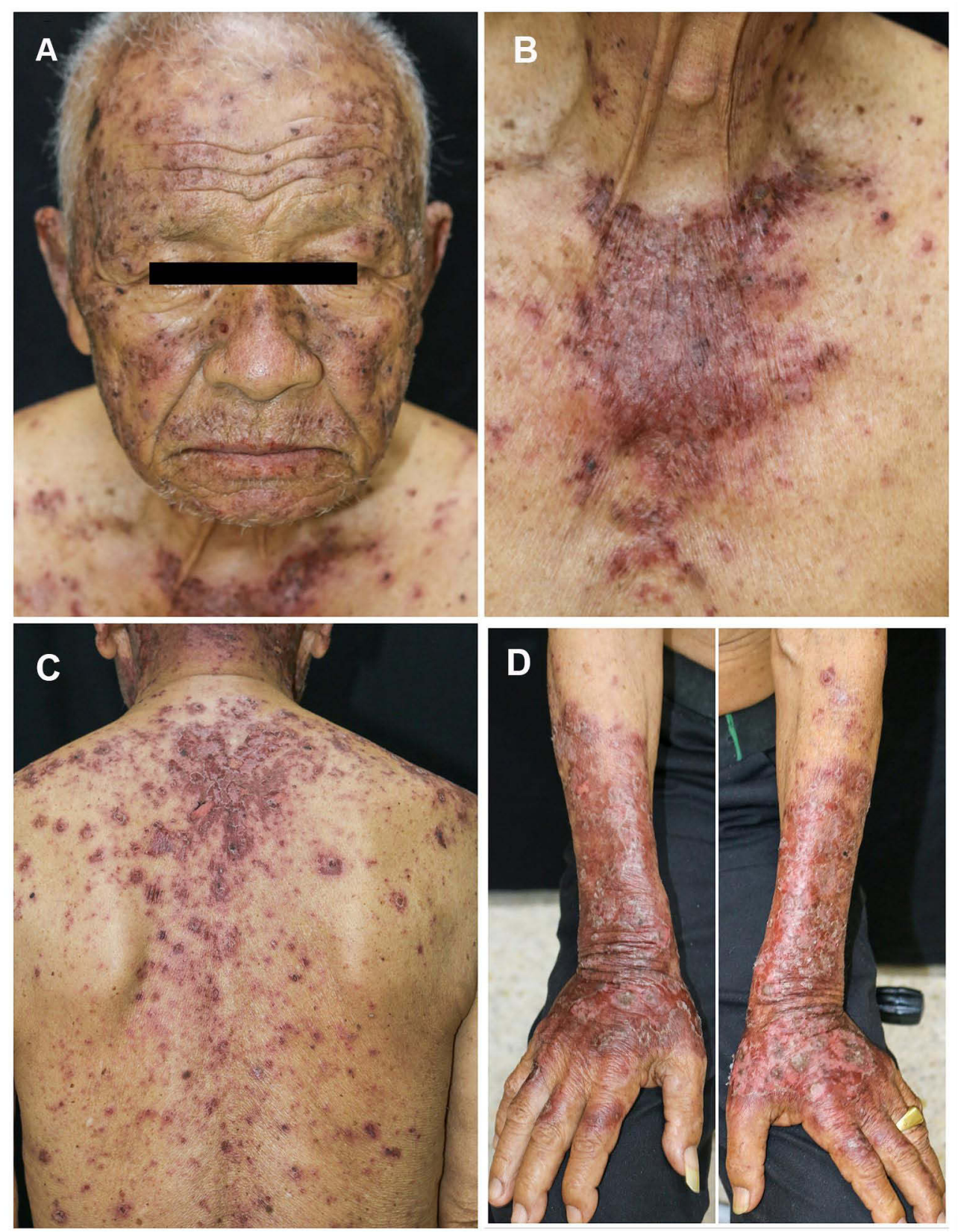

Figure I Multiple dusky red to brownish papules and patches covered by scales and crusts with some erosions predominately on face (A), upper chest (B), back (C), and dorsum of both forearms (D).

most common, accounting for $72.7 \%$ of all cases, followed by SLE with photosensitivity rash, eosinophilic fasciitis, and dermatomyositis (all 9.1\%). ${ }^{4}$ To date, atezolizumab is the only PD-L1 inhibitor associated with SCLE. Table 1 demonstrates a literature review on immune checkpoint inhibitors-associated CLE.

Durvalumab has been approved as a first-line treatment for adult patients with ES-SCLC in combination with standard-of-care chemotherapies, etoposide plus either carboplatin or cisplatin (platinum-etoposide). The regimen demonstrated a statistically significant improvement in overall survival. The most common adverse events of durvalumab (more than 20\%) in patients with ES-SCLC are neutropenia, anemia, nausea, and alopecia. $^{2}$ The increase in frequency of dermatologic toxicities has been noticed suggesting the possible association between the drug and cutaneous adverse events. The cutaneous lesions possibly associated with durvalumab consist of psoriasiform eruptions, ${ }^{5}$ cutaneous sarcoidosis, ${ }^{6}$ and dermatomyositis. $^{7}$ To the best of our knowledge, no case 


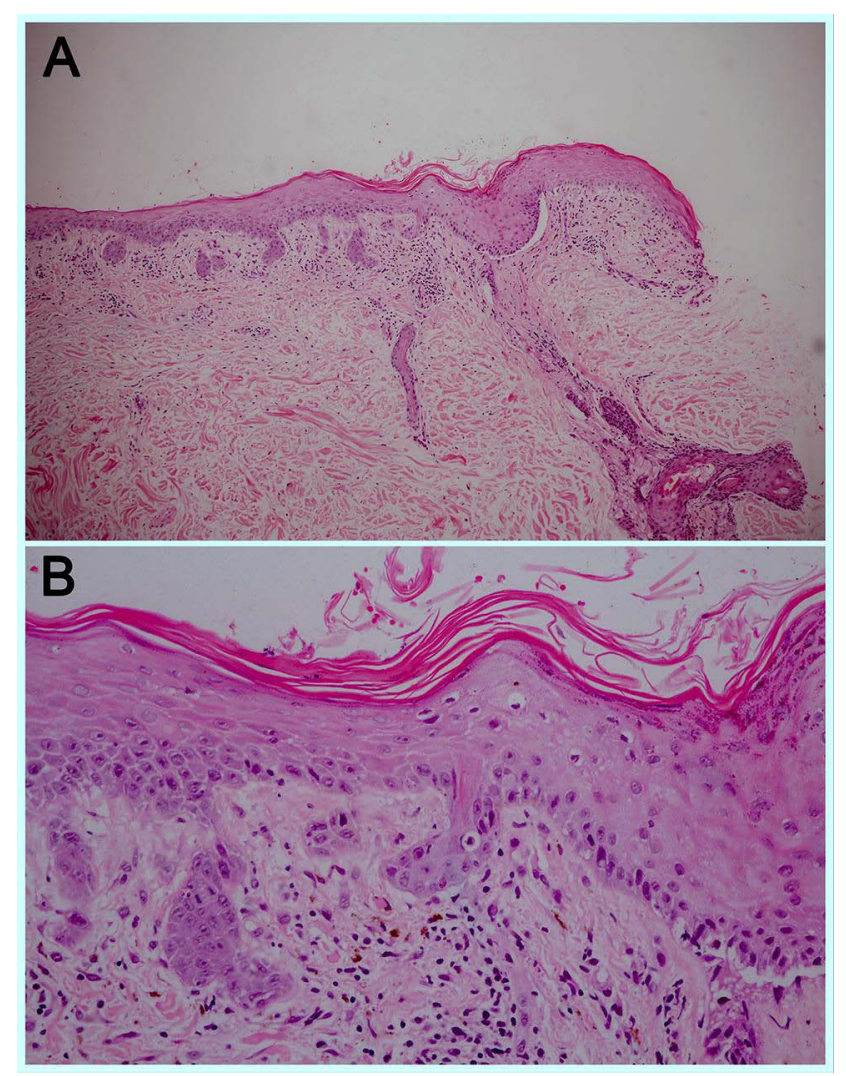

Figure 2 Superficial perivascular infiltration of lymphocytes and melanophages with marked interface change, H\&E I00X (A) Epidermal atrophy and few atypical basal keratinocytes, H\&E 400X (B).

Abbreviation: H\&E, hematoxylin and eosin.

of durvalumab-related CLE has ever been documented in the literature.

According to our case, the dusky red lesions and skin denudation can be explained by severe apoptotic injury of the epidermis, which correlates with epidermal necrosis and separation in histologic features. Interface dermatitis with foci of vacuolar alteration of basal keratinocytes alternating with areas of lichenoid dermatitis, prominent epidermal atrophy, follicular plugging, and mild or absent basement membrane thickening are all classic features of SCLE skin biopsy. ${ }^{9}$ The median time interval between drug exposure and the appearance of drug-induced SCLE (DI-SCLE) was reported to be 6 weeks (3 days to 11 years). The period of ICIs use before the onset of SCLE appears longer, averaging 4 months (2 weeks to 20 months). ${ }^{8,9}$ In our case, anti-cancer drugs consisting cytotoxic chemotherapies and durvalumab were the new medications initiated before the skin eruption in preceding 3 months and 2 months, respectively. However, DI-SCLE due to chemotherapeutic agents is uncommon, with few published cases associated with taxanes, tamoxifen, capecitabine, 5-fluorouracil, pemetrexed, and carboplatin.${ }^{10}$ There is no report on etoposide-associated SCLE in the literature. Moreover, anti-Ro/SSA can be positive in up to $80 \%$ in patients with ICIs-associated SCLE, whereas anti$\mathrm{La} / \mathrm{SSB}$ positivity occurs in only $25 \% .{ }^{9}$ The percentage of anti-Ro/SSA and ANA positivity among DI-SCLE associated with other drugs and ICIs-related SCLE were roughly the same, at $80 \%$ and $82 \%$, respectively. However, positive anti-La/SSB is more common in classic DI-SCLE, accounting for approximately $48 \% .^{8}$ Anti-histone antibodies are almost always positive in DI-SLE and patients usually lack cutaneous manifestations. Approximately one-third of the classic DI-SCLE triggers (ie, thiazide diuretics, calcium channel blockers, and terbinafine) has also been reported to be associated with antihistone antibodies; ${ }^{8}$ however, our patient had a negative result and no systemic involvement. Future studies are required to determine the frequency and pattern of LEassociated autoantibodies in ICI-related SCLE.

We conclude that the clinicopathological features, drug history and its compatible temporal relationship with rash development and positive lupus-associated serology (ie, ANA and anti-Ro/SSA) makes durvalumab associated SCLE-like eruption the most likely diagnosed. In this patient, the skin lesion resolved within one month of discontinuing immunotherapy, without recurrence after resuming the original chemotherapies.

The pathomechanism of ICIs-associated SCLE remains unclear, but many potential theories have been proposed. Firstly, the multi-hit hypothesis presumes that the PD-1 or PD-L1 blockade may unmask an immune response to a previously tolerated medication, resulting in SCLE phenotype. ${ }^{11,12}$ Another mechanism is that SCLE may be stimulated by ultraviolet (UV)-B radiation resulting in translocation of Ro (SSA) antigen and increase of cell membrane antigen expression via epitope spreading. ${ }^{13-15}$ Our patient frequently engaged in outdoor activities with inadequate photoprotection which may have aggravated the symptoms. ${ }^{16}$ Lastly, focusing on mechanism of action, anti-PD-1 or PD-L1 may modulate humoral immunity which enhances pre-existing autoantibodies and unmask latent auto-immunity. ${ }^{17-19}$ Therefore, anti-PD-L1 might be involved with immune-related adverse events, not only by increasing the activity of the immune system against antigens in both cancers and healthy tissue, but also by increasing levels of pre-existing autoantibodies. These mechanisms may explain the positive autoantibodies in our case. 

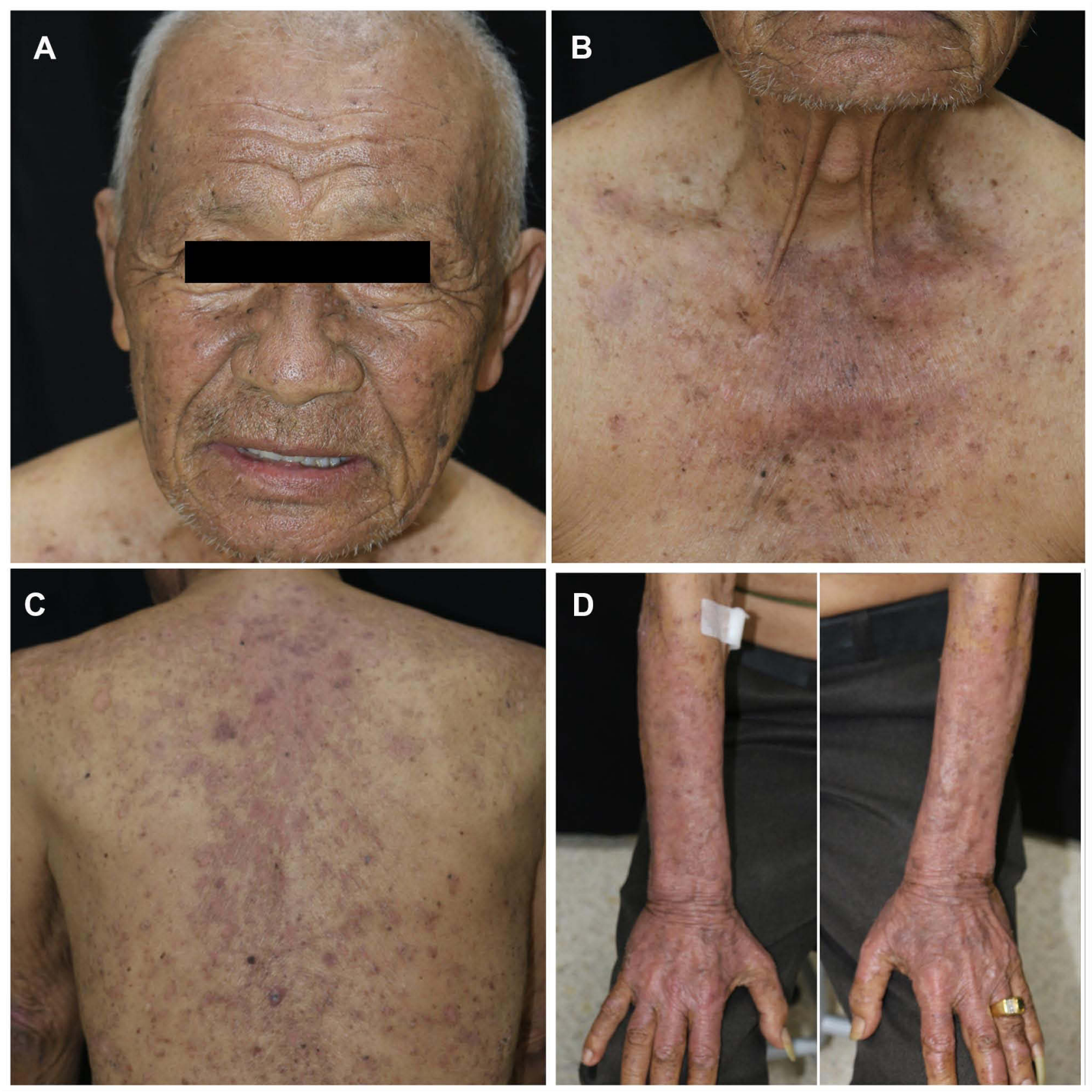

Figure 3 One month after Durvalumab was discontinued, the lesions resolved with post inflammatory hyperpigmentation on face (A), upper chest (B), back (C), and dorsum of both forearms (D).

No prospective trials have defined the best treatment approach for immune-related cutaneous adverse events. Systemic corticosteroids may be indicated for widespread or severe disease while moderate- to highpotency topical corticosteroids are first-line treatments for mild to moderate eruptions. ${ }^{3,20-22}$ In our case, the rash dramatically improved with $1 \mathrm{mg} / \mathrm{kg} /$ day of oral prednisolone. The use of antimalarial drugs has typically been recommended as the first-line systemic treatment for CLE, with response rates ranging from $31 \%$ to $91 \%{ }^{23}$ There is no strong evidence on using antimalarials in treatment for ICIs-associated SCLE. However, HCQ administered at 200-400 $\mathrm{mg}$ /day has been employed with good outcome and without any adverse events. ${ }^{9,24-28}$ In a previous study, approximately $40 \%$ showed serologic improvement at the 8 month follow-up in drug-induced CLE with positive anti-Ro/SSA. ${ }^{29}$ 
Table I Review of Reports on Checkpoint Inhibitors-Associated CLE

\begin{tabular}{|c|c|c|c|c|c|c|c|c|c|c|c|}
\hline Study & $\begin{array}{l}\text { Age } \\
\text { (yrs)/ } \\
\text { Sex }\end{array}$ & Tumor Type & $\begin{array}{l}\text { History of } \\
\text { Autoimmune } \\
\text { Disease }\end{array}$ & ICls & $\begin{array}{l}\text { Time to } \\
\text { Rash Onset }\end{array}$ & Cutaneous Manifestations & $\begin{array}{l}\text { Autoimmune } \\
\text { Serologies }\end{array}$ & Histologic/DIF Results & Treatment & $\begin{array}{l}\text { ICI } \\
\text { Interruption }\end{array}$ & $\begin{array}{l}\text { Follow- } \\
\text { Up }\end{array}$ \\
\hline Our case & $74 / \mathrm{M}$ & SCLC & None & Durvalumab & $\begin{array}{l}3 \text { infusion } \\
\text { cycles }(2 \mathrm{mo})\end{array}$ & $\begin{array}{l}\text { Multiple dusky red to brownish } \\
\text { papules and patches with scale } \\
\text { and erosions on the face, neck, } \\
\text { trunk, and extensor surface of } \\
\text { upper extremities }\end{array}$ & $\begin{array}{l}\text { ANA: I:320, } \\
\text { fine speckled } \\
\text { pattern } \\
\text { Anti-Ro (SSA): } \\
\text { positive } \\
\text { Anti-La (SSB): } \\
\text { negative } \\
\text { Anti-dsDNA: } \\
\text { negative } \\
\text { Anti-Smith: } \\
\text { negative }\end{array}$ & $\begin{array}{l}\text { H\&E: superficial perivascular infiltration, } \\
\text { epidermal atrophy with marked interface } \\
\text { change } \\
\text { Thin epidermis was necrosed and } \\
\text { separated with dysmaturation of atypical } \\
\text { basal keratinocytes } \\
\text { DIF: NA }\end{array}$ & $\begin{array}{l}\text { HCQ } 200 \mathrm{mg} / \\
\text { day, } \\
\text { prednisolone I } \\
\text { MKD, low- } \\
\text { potency topical } \\
\text { corticosteroid }\end{array}$ & $\begin{array}{l}\text { Permanently } \\
\text { discontinued }\end{array}$ & $\begin{array}{l}\text { Improved } \\
\text { within I } \\
\text { mo }\end{array}$ \\
\hline $\begin{array}{l}\text { Liu et al } \\
2018^{25}\end{array}$ & $58 / \mathrm{F}$ & NSCLC & AlHA & Nivolumab & $5 \mathrm{mo}$ & $\begin{array}{l}\text { Monomorphous, violaceous } \\
\text { papules and polycyclic, annular } \\
\text { papulosquamous plaques on the } \\
\text { back and chest }\end{array}$ & $\begin{array}{l}\text { ANA: NA } \\
\text { Anti-Ro (SSA): } \\
\text { positive } \\
\text { Anti-La (SSB): } \\
\text { NA } \\
\text { Anti-dsDNA: } \\
\text { NA } \\
\text { Anti-cardiolipin: } \\
\text { positive }\end{array}$ & $\begin{array}{l}\text { HEE: epidermal atrophy, interface } \\
\text { dermatitis with lymphocytic and } \\
\text { histiocytic infiltrate, moderate basal } \\
\text { vacuolar damage, and colloid bodies } \\
\text { DIF: negative }\end{array}$ & $\begin{array}{l}\text { HCQ } 400 \mathrm{mg} / \\
\text { day, } \\
\text { prednisolone } \\
37.5 \mathrm{mg} / \mathrm{day}, \\
\text { superpotent } \\
\text { topical } \\
\text { corticosteroid }\end{array}$ & $\begin{array}{l}\text { Discontinued } \\
\text { and restarted } 5 \\
\text { months later }\end{array}$ & Improved \\
\hline \multirow[t]{2}{*}{$\begin{array}{l}\text { Blakeway } \\
\text { et al } 2019^{20}\end{array}$} & $\begin{array}{l}\text { Case } \\
179 / \\
\mathrm{F}\end{array}$ & Melanoma & None & Pembrolizumab & $\begin{array}{l}3 \text { infusion } \\
\text { cycles }\end{array}$ & $\begin{array}{l}\text { Annular scaly rash on the face, } \\
\text { arms, torso, and legs }\end{array}$ & $\begin{array}{l}\text { ANA: negative } \\
\text { Anti-Ro (SSA): } \\
\text { NA } \\
\text { Anti-La (SSB): } \\
\text { NA } \\
\text { Anti-dsDNA: } \\
\text { NA }\end{array}$ & $\begin{array}{l}\text { H\&E: vacuolar interface pattern, colloid } \\
\text { bodies in the spinous and basal layers, and } \\
\text { moderately dense perivascular infiltrate } \\
\text { of lymphocytes in upper dermis } \\
\text { DIF: granular deposition of C3 in the } \\
\text { BMZ }\end{array}$ & $\begin{array}{l}\text { Superpotent } \\
\text { topical } \\
\text { corticosteroid }\end{array}$ & $\begin{array}{l}\text { Discontinued } \\
\text { and restarted } \\
\text { with no } \\
\text { recurrence }\end{array}$ & $\begin{array}{l}\text { Improved } \\
\text { within } 3 \\
\text { weeks }\end{array}$ \\
\hline & $\begin{array}{l}\text { Case } \\
275 / \\
M\end{array}$ & Melanoma & None & Pembrolizumab & $\begin{array}{l}9 \text { infusion } \\
\text { cycles }\end{array}$ & $\begin{array}{l}\text { Widespread, symmetrical "lupus- } \\
\text { like" dermatosis on the torso, } \\
\text { arms and legs }\end{array}$ & $\begin{array}{l}\text { ANA: negative } \\
\text { Anti-Ro (SSA): } \\
\text { NA } \\
\text { Anti-La (SSB): } \\
\text { NA } \\
\text { Anti-dsDNA: } \\
\text { NA }\end{array}$ & $\begin{array}{l}\text { H\&E: vacuolar interface pattern, colloid } \\
\text { bodies in the spinous and basal layers, } \\
\text { mildly dense perivascular infiltrate of } \\
\text { lymphocytes in upper dermis, and } \\
\text { increased dermal mucin } \\
\text { DIF: granular deposition of IgG, IgA and } \\
\text { IgM in the BMZ }\end{array}$ & $\begin{array}{l}\text { Superpotent } \\
\text { topical } \\
\text { corticosteroid }\end{array}$ & $\begin{array}{l}\text { Discontinued } \\
\text { and restarted } \\
\text { with no } \\
\text { recurrence }\end{array}$ & $\begin{array}{l}\text { Improved } \\
\text { within } 3 \\
\text { weeks }\end{array}$ \\
\hline
\end{tabular}




\begin{tabular}{|c|c|c|c|c|c|c|c|c|c|c|c|}
\hline \multirow[t]{2}{*}{$\begin{array}{l}\text { Zitouni } \\
\text { et al } 2019^{24}\end{array}$} & $\begin{array}{l}\text { Case } \\
1721 \\
\mathrm{~F}\end{array}$ & Melanoma & $\begin{array}{l}\text { Autoimmune } \\
\text { hepatitis }\end{array}$ & Nivolumab & $\begin{array}{l}13 \text { infusion } \\
\text { cycles plus } 2 \\
\text { mo after III } \\
\text { discontinuation }\end{array}$ & $\begin{array}{l}\text { Pruritic, nummular erythematous } \\
\text { plaques on the back and arms }\end{array}$ & $\begin{array}{l}\text { ANA: I:640 } \\
\text { Anti-Ro (SSA): } \\
\text { positive } \\
\text { Anti-La (SSB): } \\
\text { positive } \\
\text { Anti-dsDNA: } \\
\text { negative }\end{array}$ & $\begin{array}{l}\text { H\&E: lymphoid inflammatory infiltrates } \\
\text { predominantly in perivascular areas, and } \\
\text { focal lesions of the dermis and epidermis } \\
\text { DIF: no } C 3 \text { or lgG depositions }\end{array}$ & $\begin{array}{l}\text { HCQ } 400 \mathrm{mg} / \\
\text { day }\end{array}$ & $\begin{array}{l}\text { Permanently } \\
\text { discontinued }\end{array}$ & $\begin{array}{l}\text { Improved } \\
\text { within } 4 \\
\text { mo }\end{array}$ \\
\hline & $\begin{array}{l}\text { Case } \\
2431 \\
M\end{array}$ & NSCLC & None & Nivolumab & $\begin{array}{l}2 \text { infusion } \\
\text { cycles (I mo) }\end{array}$ & $\begin{array}{l}\text { Annular erythematous eruption } \\
\text { on the dorsal aspect of the hands, } \\
\text { arms, and chest }\end{array}$ & $\begin{array}{l}\text { ANA: I:320 } \\
\text { Anti-Ro (SSA): } \\
>600 \text { IU/mL } \\
\text { Anti-La (SSB): } \\
\text { NA } \\
\text { Anti-dsDNA: } \\
\text { NA }\end{array}$ & $\begin{array}{l}\text { HRE: discrete Iymphoid perivascular } \\
\text { inflammatory infiltrates } \\
\text { DIF: scarce C3 deposits along the BMZ }\end{array}$ & $\begin{array}{l}\text { HCQ } 400 \mathrm{mg} / \\
\text { day, } \\
\text { prednisolone I } \\
\text { MKD, potent } \\
\text { topical } \\
\text { corticosteroid }\end{array}$ & $\begin{array}{l}\text { Permanently } \\
\text { discontinued }\end{array}$ & $\begin{array}{l}\text { Severe } \\
\text { flare-up } \\
\text { after 2 } \\
\text { weeks of } \\
\text { the } \\
\text { treatment }\end{array}$ \\
\hline $\begin{array}{l}\text { Kosche } \\
\text { et al } 2019^{26}\end{array}$ & $75 / F$ & $\begin{array}{l}\text { Serous ovarian } \\
\text { cancer }\end{array}$ & None & $\begin{array}{l}\text { Ipilimumab and } \\
\text { Nivolumab }\end{array}$ & $\begin{array}{l}2 \text { infusion } \\
\text { cycles }\end{array}$ & $\begin{array}{l}\text { Pruritic, erychematous, red- } \\
\text { brown, scaly plaques with an } \\
\text { arcuate appearance on the back, } \\
\text { abdomen, arms, and legs }\end{array}$ & $\begin{array}{l}\text { ANA: I:160, } \\
\text { speckled } \\
\text { pattern } \\
\text { Anti-Ro (SSA): } \\
>8.0 \\
\text { Anti-La (SSB): } \\
\text { negative } \\
\text { AntidsDNA: } \\
\text { negative } \\
\text { Anti-Smith: } \\
\text { negative }\end{array}$ & $\begin{array}{l}\text { HEE: interface lymphocytic infiltrate and } \\
\text { focal basal vacuolar change } \\
\text { DIF: NA }\end{array}$ & $\begin{array}{l}\text { HCQ } 400 \mathrm{mg} / \\
\text { day, quinacrine } \\
100 \mathrm{mg} / \text { day, } \\
\text { prednisone } 40 \\
\text { mg/day, mid- } \\
\text { strength topical } \\
\text { corticosteroid }\end{array}$ & $\begin{array}{l}\text { Discontinued } \\
\text { and switched } \\
\text { to } \\
\text { pembrolizumab }\end{array}$ & $\begin{array}{l}\text { Improved } \\
\text { within I } \\
\text { week and } \\
\text { later } \\
\text { flared up }\end{array}$ \\
\hline $\begin{array}{l}\text { Ogawa- } \\
\text { Momohara } \\
\text { et al } 2020^{22}\end{array}$ & 80/M & Melanoma & None & Pembrolizumab & $\begin{array}{l}5 \text { infusion } \\
\text { cycles }\end{array}$ & $\begin{array}{l}\text { Multiple annular erythema on the } \\
\text { trunk }\end{array}$ & $\begin{array}{l}\text { ANA: NA } \\
\text { Anti-Ro (SSA): } \\
\text { positive } \\
\text { Anti-La (SSB): } \\
\text { NA } \\
\text { Anti-dsDNA: } \\
\text { negative }\end{array}$ & $\begin{array}{l}\text { H\&E: strong liquefaction degeneration } \\
\text { and dense superficial dermal and } \\
\text { perivascular Iymphocytic infiltration } \\
\text { DIF: no C3 or IgG depositions }\end{array}$ & $\begin{array}{l}\text { Prednisolone I } \\
\text { MKD, topical } \\
\text { corticosteroid }\end{array}$ & $\begin{array}{l}\text { Discontinued } \\
\text { at the } 9 \text { th cycle }\end{array}$ & $\begin{array}{l}\text { Improved } \\
\text { within } 3 \\
\text { mo }\end{array}$ \\
\hline
\end{tabular}

(Continued) 
Table I (Continued).

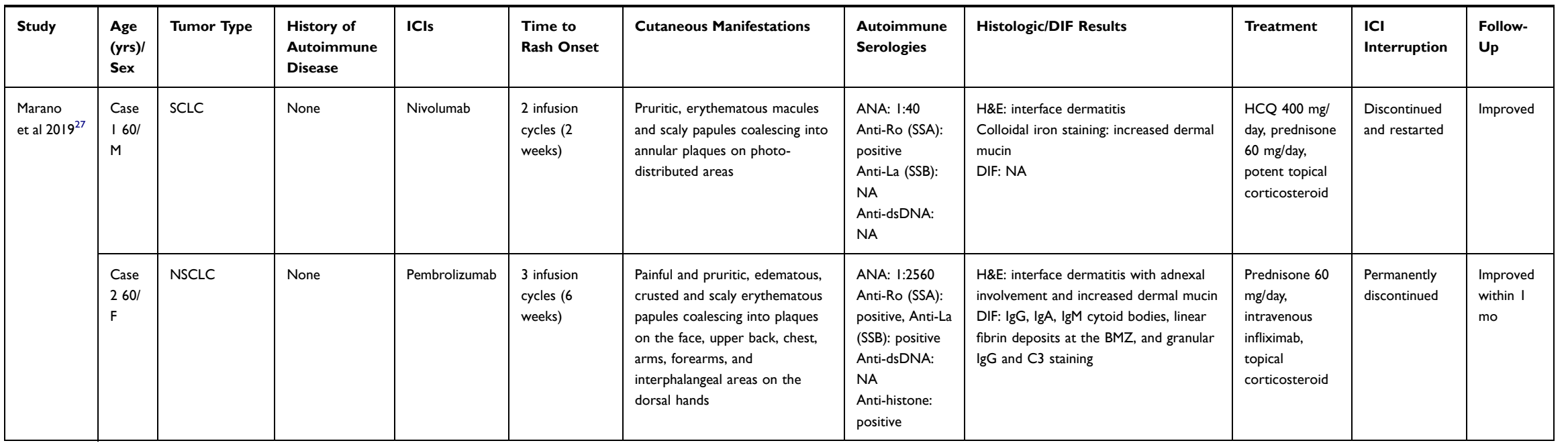




\begin{tabular}{|c|c|c|c|c|c|c|c|c|c|c|c|}
\hline \multirow[t]{5}{*}{$\begin{array}{l}\text { Bui et al } \\
2021^{9}\end{array}$} & $\begin{array}{l}\text { Case } \\
154 / \\
\text { F }\end{array}$ & SCLC & $\begin{array}{l}\text { ICl-associated } \\
\text { psoriasis }\end{array}$ & Nivolumab & $20 \mathrm{mo}$ & $\begin{array}{l}\text { Annular eruption on the trunk } \\
\text { and extremities }\end{array}$ & $\begin{array}{l}\text { ANA: I:5120, } \\
\text { speckled } \\
\text { pattern } \\
\text { Anti-Ro (SSA): } \\
>8.0 \\
\text { Anti-La (SSB): } \\
>8.0 \\
\text { anti-dsDNA: } \\
\text { negative }\end{array}$ & $\begin{array}{l}\text { H\&E: focal interface dermatitis, focal } \\
\text { lichenoid dermal lymphocytes infiltrate, } \\
\text { and mild dermal mucin deposition } \\
\text { DIF: NA }\end{array}$ & $\begin{array}{l}\text { HCQ } 200 \mathrm{mg} / \\
\text { day, potent } \\
\text { topical } \\
\text { corticosteroid }\end{array}$ & $\begin{array}{l}\text { Continued } \\
\text { with no } \\
\text { interruption }\end{array}$ & $\begin{array}{l}\text { Complete } \\
\text { clearance } \\
\text { within } 6 \\
\text { mo }\end{array}$ \\
\hline & $\begin{array}{l}\text { Case } \\
254 / \\
\text { F }\end{array}$ & Ovarian cancer & None & PD-I inhibitor & $4 \mathrm{mo}$ & $\begin{array}{l}\text { Annular eruption on the upper } \\
\text { extremities and trunk }\end{array}$ & $\begin{array}{l}\text { ANA: negative } \\
\text { Anti-Ro (SSA): } \\
\text { negative } \\
\text { Anti-La (SSB): } \\
\text { negative } \\
\text { Anti-dsDNA: } \\
\text { negative }\end{array}$ & $\begin{array}{l}\text { H\&E: interface dermatitis, epidermal } \\
\text { spongiosis, superficial dermal perivascular } \\
\text { lymphocytes infiltrate with rare } \\
\text { eosinophils, follicular plugging and subtle } \\
\text { dermal mucin deposition } \\
\text { DIF: granular C3, IgM, and IgG along the } \\
\text { BMZ }\end{array}$ & $\begin{array}{l}\text { Potent topical } \\
\text { corticosteroid }\end{array}$ & $\begin{array}{l}\text { Discontinued } \\
\text { and restarted I } \\
\text { month later }\end{array}$ & $\begin{array}{l}\text { Improved } \\
\text { within } 2 \\
\text { mo }\end{array}$ \\
\hline & $\begin{array}{l}\text { Case } \\
357 / \\
F\end{array}$ & Breast cancer & $\begin{array}{l}\text { ICl-associated } \\
\text { Sjogren's } \\
\text { syndrome }\end{array}$ & Atezolizumab & $11.5 \mathrm{mo}$ & $\begin{array}{l}\text { Annular eruption on the upper } \\
\text { extremities and trunk }\end{array}$ & $\begin{array}{l}\text { ANA: I:320, } \\
\text { speckled } \\
\text { pattern } \\
\text { Anti-Ro (SSA): } \\
>8.0 \\
\text { Anti-La (SSB): } \\
\text { negative } \\
\text { Anti-dsDNA: } \\
\text { negative }\end{array}$ & $\begin{array}{l}\text { H\&E: interface dermatitis, focal lichenoid } \\
\text { infiltrate, superficial to mid-dermal } \\
\text { perivascular lymphocytic infiltrate, } \\
\text { perifollicular plugging and increased } \\
\text { dermal mucin deposition } \\
\text { DIF: negative }\end{array}$ & $\begin{array}{l}\text { Superpotent } \\
\text { topical } \\
\text { corticosteroid }\end{array}$ & $\begin{array}{l}\text { Permanently } \\
\text { discontinued } 2 \\
\text { mo prior to } \\
\text { rash onset for } \\
\text { colitis }\end{array}$ & $\begin{array}{l}\text { Improved } \\
\text { within I } \\
\text { mo }\end{array}$ \\
\hline & $\begin{array}{l}\text { Case } \\
465 / \\
M\end{array}$ & SCLC & None & Pembrolizumab & $3 \mathrm{mo}$ & $\begin{array}{l}\text { Eruption on the trunk and } \\
\text { extremities }\end{array}$ & $\begin{array}{l}\text { ANA: I: } 320, \\
\text { speckled } \\
\text { pattern } \\
\text { Anti-Ro (SSA): } \\
>8.0 \\
\text { Anti-La (SSB): } \\
\text { negative } \\
\text { Anti-dsDNA: } \\
\text { NA }\end{array}$ & $\begin{array}{l}\text { H\&E: prominent interface dermatitis, } \\
\text { focal vesicle formation, lichenoid } \\
\text { infiltrate, prominent dyskeratotic } \\
\text { keratinocytes with epidermal necrosis, } \\
\text { and superficial to mid-dermal } \\
\text { perivascular, periadnexal lymphocytic } \\
\text { infiltrate and follicular plugging } \\
\text { DIF: negative }\end{array}$ & $\begin{array}{l}\text { HCQ } 400 \mathrm{mg} / \\
\text { day, potent } \\
\text { topical } \\
\text { corticosteroid }\end{array}$ & $\begin{array}{l}\text { Permanently } \\
\text { discontinued }\end{array}$ & $\begin{array}{l}\text { Improved } \\
\text { within } 2 \\
\text { mo }\end{array}$ \\
\hline & $\begin{array}{l}\text { Case } \\
560 / \\
M\end{array}$ & Melanoma & None & Nivolumab & $0.5 \mathrm{mo}$ & $\begin{array}{l}\text { Annular eruption on the } \\
\text { extremities and trunk }\end{array}$ & $\begin{array}{l}\text { ANA: I:320, } \\
\text { speckled } \\
\text { pattern } \\
\text { Anti-Ro (SSA): } \\
>8.0 \\
\text { Anti-La (SSB): } \\
\text { negative } \\
\text { Anti-dsDNA: } \\
\text { NA }\end{array}$ & $\begin{array}{l}\text { H\&E: prominent interface dermatitis, } \\
\text { lichenoid infiltrate, clefting, prominent } \\
\text { superficial to deep dermal perivascular, } \\
\text { periadnexal lymphocytic infiltrate and } \\
\text { increased dermal mucin deposition } \\
\text { DIF: negative }\end{array}$ & $\begin{array}{l}\text { Potent topical } \\
\text { corticosteroid }\end{array}$ & $\begin{array}{l}\text { Continued } \\
\text { with no } \\
\text { interruption }\end{array}$ & $\begin{array}{l}\text { Improved } \\
\text { within } 2 \\
\text { mo }\end{array}$ \\
\hline
\end{tabular}

(Continued) 
Table I (Continued).

\begin{tabular}{|c|c|c|c|c|c|c|c|c|c|c|c|}
\hline Study & $\begin{array}{l}\text { Age } \\
\text { (yrs)/ } \\
\text { Sex }\end{array}$ & Tumor Type & $\begin{array}{l}\text { History of } \\
\text { Autoimmune } \\
\text { Disease }\end{array}$ & ICls & $\begin{array}{l}\text { Time to } \\
\text { Rash Onset }\end{array}$ & Cutaneous Manifestations & $\begin{array}{l}\text { Autoimmune } \\
\text { Serologies }\end{array}$ & Histologic/DIF Results & Treatment & $\begin{array}{l}\text { ICI } \\
\text { Interruption }\end{array}$ & $\begin{array}{l}\text { Follow- } \\
\text { Up }\end{array}$ \\
\hline $\begin{array}{l}\text { Takeda } \\
\text { et al } 2021^{28}\end{array}$ & 49/F & $\begin{array}{l}\text { Oropharyngeal } \\
\text { carcinoma }\end{array}$ & None & Pembrolizumab & 2 weeks & $\begin{array}{l}\text { Persistent erythema, and purple } \\
\text { discoloration on the fingers, } \\
\text { lower legs, and feet }\end{array}$ & $\begin{array}{l}\text { ANA: l:1280, } \\
\text { speckled } \\
\text { pattern } \\
\text { Anti-Ro (SSA): } \\
\text { positive } \\
\text { Anti-La (SSB): } \\
\text { NA } \\
\text { Anti-dsDNA: } \\
\text { negative } \\
\text { Anti-Smith: } \\
\text { positive } \\
\text { Anti- } \\
\text { phospholipid: } \\
\text { negative } \\
\text { ANCA: } \\
\text { negative } \\
\text { Cryoglobulin: } \\
\text { negative } \\
\text { C3: Low, C4: } \\
\text { normal }\end{array}$ & NA & $\begin{array}{l}\text { HCQ } 200 \mathrm{mgl} \\
\text { day, } \\
\text { prednisolone } 30 \\
\text { mg/day }\end{array}$ & NA & NA \\
\hline
\end{tabular}
female; HCQ, hydroxychloroquine; H\&E, hematoxylin and eosin stain; ICls, immune checkpoint inhibitors; IU/mL, International Unit/mL; M, male; MKD, mg/kg/day; mo, months; NA, not available; NSCLC, non-small cell lung cancer. 
In conclusion, ICIs are increasingly used in cancer therapy, the clinical-pathologic correlation is important to diagnose specific dermatologic adverse events. Prospective trials are required to define the best treatment approaches and recommendations for minimizing immunotherapy interruption and avoiding immunosuppressive medications whenever possible.

\section{Consent Statement}

Written informed consent was provided by the patient to have the case details and any accompanying images published. Institutional approval was not required to publish the case details.

\section{Funding}

Division of Dermatology, Faculty of Medicine, Ramathibodi Hospital, Mahidol University, Bangkok, Thailand.

\section{Disclosure}

The authors report no conflicts of interest in this work.

\section{References}

1. Postow MA, Sidlow R, Hellmann MD. Immune-related adverse events associated with immune checkpoint blockade. N Engl J Med. 2018;378 (2):158-168. doi:10.1056/NEJMra1703481

2. Paz-Ares L, Dvorkin M, Chen Y, et al. Durvalumab plus platinumetoposide versus platinum-etoposide in first-line treatment of extensive-stage small-cell lung cancer (CASPIAN): a randomised, controlled, open-label, Phase 3 trial. Lancet. 2019;394(10212):19291939. doi:10.1016/s0140-6736(19)32222-6

3. Geisler AN, Phillips GS, Barrios DM, et al. Immune checkpoint inhibitor-related dermatologic adverse events. $J$ Am Acad Dermatol. 2020;83(5):1255-1268. doi:10.1016/j.jaad.2020.03.132

4. Bui AN, Singer S, Hirner J, et al. De novo cutaneous connective tissue disease temporally associated with immune checkpoint inhibitor therapy: a retrospective analysis. $J$ Am Acad Dermatol. 2021;84(3):864 869. doi:10.1016/j.jaad.2020.10.054

5. Lin WH, Lee KY, Lee WR, Shih YH. Durvalumab-induced de novo annular psoriasiform drug eruption successfully treated with a combination of narrowband ultraviolet B phototherapy and topical treatment. $J$ Dermatol. 2020;47(9):1041-1045. doi:10.1111/1346-8138.15371

6. Rousseau PM, Raimbourg J, Robert M, Dansette D, Dréno B, Peuvrel L. First case of cutaneous sarcoidosis within tattoos under durvalumab. Int J Dermatol. 2019;58(9):e168-e170. doi:10.1111/ijd.14484

7. Coustal C, Du Thanh A, Roubille F, Assenat E, Maria ATJ. Rare cutaneous toxicity of immune checkpoint inhibitors: a case of durvalumab-induced dermatomyositis. Eur $J$ Cancer. 2021;155:25-27. doi:10.1016/j.ejca.2021.06.031

8. Lowe GC, Henderson CL, Grau RH, Hansen CB, Sontheimer RD. A systematic review of drug-induced subacute cutaneous lupus erythematosus. Br J Dermatol. 2011;164(3):465-472. doi:10.1111/j.13652133.2010.10110.x

9. Bui AN, Hirner J, Singer SB, et al. De novo subacute cutaneous lupus erythematosus-like eruptions in the setting of programmed death-1 or programmed death ligand-1 inhibitor therapy: clinicopathological correlation. Clin Exp Dermatol. 2021;46(2):328-337. doi:10.1111/ ced. 14449
10. González García A, Sifuentes Giraldo WA, Grillo Fernández E, Zea Mendoza A. Subacute cutaneous lupus erythematosus associated with pemetrexed plus Carboplatin chemotherapy. J Clin Rheumatol. 2014;20(8):449-450. doi:10.1097/rhu.0000000000000183

11. Lin JH, Dutz JP, Sontheimer RD, Werth VP. Pathophysiology of cutaneous lupus erythematosus. Clin Rev Allergy Immunol. 2007;33 (1-2):85-106. doi:10.1007/s12016-007-0031-x

12. Shi VJ, Rodic N, Gettinger S, et al. Clinical and histologic features of lichenoid mucocutaneous eruptions due to anti-programmed cell death 1 and anti-programmed cell death ligand 1 immunotherapy. JAMA Dermatol. 2016;152(10):1128-1136. doi:10.1001/jamad ermatol.2016.2226

13. Vanderlugt CL, Miller SD. Epitope spreading in immune-mediated diseases: implications for immunotherapy. Nat Rev Immunol. 2002;2 (2):85-95. doi:10.1038/nri724

14. Chanprapaph K, Limtong P, Ngamjanyaporn P, Suchonwanit P. Trichoscopic signs in dermatomyositis, systemic lupus erythematosus, and systemic sclerosis: a comparative study of 150 patients. Dermatology. 2021;1-11. doi:10.1159/000520297

15. Wiznia LE, Subtil A, Choi JN. Subacute cutaneous lupus erythematosus induced by chemotherapy: gemcitabine as a causative agent. JAMA Dermatol. 2013;149(9):1071-1075. doi:10.1001/ jamadermatol.2013.4957

16. Chanprapaph K, Ploydaeng M, Pakornphadungsit K, Mekwilaiphan T, Vachiramon V, Kanokrungsee S. The behavior, attitude, and knowledge towards photoprotection in patients with cutaneous/systemic lupus erythematosus: a comparative study with 526 patients and healthy controls. Photochem Photobiol Sci. 2020;19(9):12011210. doi:10.1039/d0pp00073f

17. Hasan Ali O, Bomze D, Ring SS, et al. BP180-specific IgG is associated with skin adverse events, therapy response, and overall survival in non-small cell lung cancer patients treated with checkpoint inhibitors. $J$ Am Acad Dermatol. 2020;82(4):854-861. doi:10.1016/j.jaad.2019.08.045

18. Seree-aphinan C, Chanprapaph K, Rattanakaemakorn P, et al. Inactivated COVID-19 Vaccine Induces a Low Humoral Immune Response in a Subset of Dermatological Patients Receiving Immunosuppressants. Front Med. 2021;8:769845. doi:10.3389/ fmed.2021.769845

19. Osorio JC, Ni A, Chaft JE, et al. Antibody-mediated thyroid dysfunction during T-cell checkpoint blockade in patients with non-small-cell lung cancer. Ann Oncol. 2017;28(3):583-589. doi:10.1093/annonc/ mdw640

20. Blakeway EA, Elshimy N, Muinonen-Martin A, Marples M, Mathew B, Mitra A. Cutaneous lupus associated with pembrolizumab therapy for advanced melanoma: a report of three cases. Melanoma Res. 2019;29(3):338-341. doi:10.1097/cmr.0000000000000587

21. Chanprapaph K, Pomsoong C, Kositkuljorn C, Suchonwanit P. Intramuscular corticosteroid therapy in the treatment of alopecia areata: a time-to-event analysis. Drug Des Devel Ther. 2022;16:107-116. doi:10.2147/DDDT.S342179

22. Ogawa-Momohara M, Muro Y, Goto K, et al. Subacute cutaneous lupus erythematosus with melanocyte elimination induced by pembrolizumab. J Dermatol. 2020;47(6):e217-e219. doi:10.1111/13468138.15316

23. Chasset F, Bouaziz JD, Costedoat-Chalumeau N, Francès C, Arnaud L. Efficacy and comparison of antimalarials in cutaneous lupus erythematosus subtypes: a systematic review and meta-analysis. $\mathrm{Br}$ J Dermatol. 2017;177(1):188-196. doi:10.1111/bjd.15312

24. Zitouni NB, Arnault JP, Dadban A, Attencourt C, Lok CC, Chaby G. Subacute cutaneous lupus erythematosus induced by nivolumab: two case reports and a literature review. Melanoma Res. 2019;29(2):212215. doi: $10.1097 / \mathrm{cmr} .0000000000000536$

25. Liu RC, Sebaratnam DF, Jackett L, Kao S, Lowe PM. Subacute cutaneous lupus erythematosus induced by nivolumab. Australas $J$ Dermatol. 2018;59(2):e152-e154. doi:10.1111/ajd.12681 
26. Kosche C, Owen JL, Choi JN. Widespread subacute cutaneous lupus erythematosus in a patient receiving checkpoint inhibitor immunotherapy with ipilimumab and nivolumab. Dermatol Online J. 2019;25(10). doi:10.5070/D32510045821

27. Marano AL, Clarke JM, Morse MA, et al. Subacute cutaneous lupus erythematosus and dermatomyositis associated with anti-programmed cell death 1 therapy. $B r \quad J$ Dermatol. 2019;181(3):580-583. doi:10.1111/bjd.17245
28. Takeda K, Nakano K, Udagawa S, et al. Chilblain lupus-like cutaneous reaction associated with systemic lupus erythematosus induced by immune checkpoint inhibitor. Rheumatology. 2021. doi:10.1093/ rheumatology/keab670

29. Srivastava M, Rencic A, Diglio G, et al. Drug-induced, Ro/SSApositive cutaneous lupus erythematosus. Arch Dermatol. 2003;139 (1):45-49. doi:10.1001/archderm.139.1.45

\section{Publish your work in this journal}

Clinical, Cosmetic and Investigational Dermatology is an international, peer-reviewed, open access, online journal that focuses on the latest clinical and experimental research in all aspects of skin disease and cosmetic interventions. This journal is indexed on CAS.
The manuscript management system is completely online and includes a very quick and fair peer-review system, which is all easy to use. Visit http://www.dovepress.com/testimonials.php to read real quotes from published authors. 